\title{
Anthós
}

\section{Materialist and Causal Bridges Over the Explanatory Gap}

Celine Geday

Portland State University

Follow this and additional works at: https://pdxscholar.library.pdx.edu/anthos

Part of the Psychology Commons Let us know how access to this document benefits you.

\section{Recommended Citation}

Geday, Celine (2017) "Materialist and Causal Bridges Over the Explanatory Gap," Anthós: Vol. 8: Iss. 1, Article 8.

https://doi.org/10.15760/anthos.2017.95

This open access Article is distributed under the terms of the Creative Commons Attribution-NonCommercialShareAlike 4.0 International License (CC BY-NC-SA 4.0). All documents in PDXScholar should meet accessibility standards. If we can make this document more accessible to you, contact our team. 


\section{Materialist and Causal Bridges Over the Explanatory Gap}

Celine Geday

\section{Introduction: The Explanatory Gap}

The explanatory gap about the subjective nature of conscious experience is the gap in explanation between conscious experience, and available scientific explanations of conscious experience. The explanatory gap is, as Joseph Levine states, the problem that physicalism (or the physical sciences) has in describing mental terms and experiences. ${ }^{7}$ Thomas Nagel also describes the explanatory gap problem as the problem of explaining the subjective in objective terms. He states that the subjective viewpoint of the individual is inherently at odds with an objective, or physical explanation. ${ }^{8}$ Describing someone's experience of the redness of red, for example, by describing the neural mechanisms that fire when he or she sees red might seem to leave out the phenomenal aspect of experience in explanation. This is an example of what might be thought of as a gap in explanation between what the physical sciences say about mental properties, and what introspection reveals about mental properties. Describing mental events as identical with brain events is normally called materialism, which stands in opposition to a dualist, or non-reductivist treatment of the psychological nature of mental phenomena.

\footnotetext{
7 Joseph Levine, “On Leaving Out What It's Like," in The Nature of Consciousness: Philosophical Debates, ed. Ned Block, Owen Flanagan, Güven Güzeldere. (Cambridge: The MIT Press, 1997), 547-548, 550.

8 Thomas Nagel, "What Is It Like to Be a Bat?" The Philosophical Review 83 (1974): 442-443, 448.
} 
I offer here some arguments to motivate the materialist side of the explanatory gap problem. The argument that I believe provides the greatest impetus for a materialist understanding of mental events is that of the causal closure of the physical world. I present this argument through the lens of Jaegwon Kim's work. The causal closure of the physical world can be thought of as the completeness of physical theories, with no epiphenomenal residue supervening on, or existing over-and-above physical properties. Epiphenomenal properties are those that have no causal effect on the world; they are causally inert. Because they are causally inert, it remains difficult to see how they can be explained by physically-explainable phenomena, being nonphysical themselves. The causal closure of the physical world, I suggest, is best illuminated with respect to the problem of the gaps in explanations of consciousness through the work of Jaegwon Kim. This kind of argument is also known in metaphysics as the "Eleatic Principle" originating from Graham Oddie's work, which in part, refers to Plato's Eleatic Stranger in the Sophist. I argue that theories of qualitative properties must cohere with the causal closure of the physical world, and so must meet a causal closure condition. I use the term qualitative properties to refer to the properties of mental states that can then refer to the character of sensations, which is taken from Austen Clark. ${ }^{9}$ Qualitative properties, for Clark, are properties of sensation that enable the phenomenal appearances of things. ${ }^{10} \mathrm{On}$ Clark's model, sensations enable phenomenal experience, so this term

\footnotetext{
${ }^{9}$ Austen Clark, A Theory of Sentience, (Oxford: Oxford University Press, 2000), 8, http://selfpace.uconn.edu/paper/ClarkAusSentient.pdf.

${ }^{10}$ Ibid., 6. 
will be consistent with a materialist description of

subjective experience.

I then offer what I take to be the best materialist account available that is likely to be compatible with a causal closure condition. This account is Austen Clark's feature-placing hypothesis. Although this account is compatible with Kim's causal closure, it does complicate Kim's secondary conclusion from an argument for causal closure, which is that qualia should be defined functionally, by the roles that they perform. Feature-placing is at odds with functionalism because feature-placing defines qualitative properties relationally, without any direct reference to distal, causal stimuli. Kim puts forward functionalism as a way of individuating different qualia uniquely, however Clark's features themselves are not defined by their causal role, but relationally. In this way, features and organisms' abilities to locate them involve no direct reference to distal properties. Clark suggests that features are relational and not functionally definable by the distal properties that directly might cause them, and he bases this idea on two notions. The first is that there is wide variation across observers, such that reference to a single stimulus that causes sensation of orange does not account for differences in observers. ${ }^{11}$ The second is that there remain problems of individuating qualitative properties, in reference to stimuli, uniquely for a functionalist account. ${ }^{12}$ The idea that one observer might see the color green for what another person sees as red illustrates the problem, and

\footnotetext{
11 Ibid., 12.

12 Austen Clark, "Sensing, Objects, and Awareness: Reply to Commentators," Philosophical Psychology 17 (2010): 577.
} 
this is known as the problem of inverted qualia. Clark argues hence, that what makes qualitative properties unique cannot be defined by stimuli, ${ }^{13}$ but rather by picking out the locations of qualitative properties in space-time.

Despite Clark's account being at odds with functionalism, the feature-placing hypothesis provides a viable demonstration of the individuation of qualitative properties in another way, which is that of positing the spatial discrimination capacities of organisms, and these capacities are enabled by neural functions. He demonstrates that we have spatial discrimination capacities to pick out the locations of features. Spatial discrimination capacities are enabled by cortical maps in the brain, whose information, when bound together by the subject, allows for the individuation of unique qualia, and allows for the relational content of qualitative properties themselves to refer to their locations in space around a perceiver.

Clark also suggests that on the feature-placing hypothesis, sentience is a primitive stage of perception, within a hierarchy of preattentive processes leading up to the conscious awareness of an organism. Clark holds that we have both spatial discrimination capacities, and capacities to discriminate qualitative properties, or the features of sensation. These capacities used together serve to individuate the content of qualia uniquely.

Clark's account remains compatible with causal closure because feature-placing individuates qualia uniquely through the spatial discrimination capacities that organisms possess, and those

\footnotetext{
${ }^{13}$ Clark, A Theory of Sentience, 12. 98
} 
capacities are neural functions. This aspect of Clark's hypothesis is compatible with the idea that mental properties do not supervene on physical properties, but rather, are physical processes. This is how Clark's account remains compatible with a causal closure condition, in that the spatial discrimination capacities of perceivers reduce to properties of brain mechanisms, such as neural feature maps. Feature maps are cortical maps that guide behavior. ${ }^{[14][15]}$ Clark's featureplacing hypothesis also remains compatible with causal closure because it is preattentive- that is, it is an account of early perceptual processes and thus sensory content on this account, is nonconceptual. I offer that both Kim's and Clark's accounts support physical explanations of qualia and motivate the materialist side of the explanatory gap.

In conclusion, I offer that Clark's account of spatial discrimination and relational qualia is compatible with the causal closure of the physical world because the processes of feature-placing are reducible to neural states. I present Kim's causal closure argument because I believe it is the strongest motivation for physical explanations of mental content. I argue that explaining qualitative properties relationally, and explaining their individuation through spatial discrimination is the best way to approach the problem of solving the explanatory gap in that it provides a potential bridge from consciousness to physical theories that is compatible with naturalism.

\footnotetext{
14 Ibid., 45.

15 Austen Clark, "How Do Feature Maps Represent?" Early Content Conference (2006): http://selfpace.uconn.edu/paper/ClarkAusUmEarly.html
} 


\section{Jaegwon Kim and Mental Causation}

Jaegwon Kim in his book Physicalism, or Something Near Enough proposes that mental causation cannot occur unless the mental is physically reducible. ${ }^{16} \mathrm{Kim}$ offers that the only way to reduce aspects of consciousness, like sensation to physical states is by functionally explaining the processes that constitute an individual's sensory experiences. ${ }^{17} \mathrm{~A}$ functional description of the mechanisms involved in sensation will iterate the jobs that those mechanisms have in the system of the organism. For example, on a sort of low-level or realizer functionalist position, a pain sensation can be described as being identical with C-fibers firing in in an organism's nervous system. In this way, Kim takes an ontological naturalist standpoint in which there are no immaterial substances in the world like souls, or immaterial minds. ${ }^{[18][19]}$ This can also be thought of broadly as the notion that world is composed of physical parts, whatever those parts may be.

Theories of consciousness for Kim, and thus the causal status of the mental, need to be based on the causal closure of physical world. ${ }^{20}$ The causal closure of the physical world on Kim's analysis states that "if a physical event at time $t$ has a cause at $t$, then it has a

\footnotetext{
${ }^{16}$ Jaegwon Kim, Physicalism, or Something Near Enough (Princeton \& Oxford: Princeton University Press, 2005), 159.

${ }^{17}$ Ibid., 172.

${ }^{18}$ Ibid., 71, 84.

19 Jaegwon Kim, "From Naturalism to Physicalism: Supervenience Redux,"

Proceeding and Addresses of the American Philosophical Association 85 (2011): 113.

${ }^{20}$ Kim, Physicalism, or Something Near Enough, 156. 100
} 
physical cause at time $t .{ }^{.21}$ The consequence of Kim's analysis of mental causation is that the subjective character of experience loses its causal effectiveness. Kim states that traditional emergentist, or supervenience theories (which are non-reductive physicalist theories about the mental) take the position that the mental is both irreducible to the physical, and has causal efficacy. ${ }^{22}$ Kim states that this form of mind-body supervenience is a way of saving agency and free will. ${ }^{23}$ Emergentist and supervenience theories believe the mental to be irreducible to the physical because consciousness is said to have intrinsic and ineffable properties. ${ }^{24}$ However, Kim states that these theories face the problem of overdetermination. ${ }^{25}$ In this way, those theories are incompatible with causal closure. For example, consider the scenario in which there are two (supposedly) causal properties for one effect that is physical. If one of the causal properties is physical and the other is non-physical, in determining which of these caused the effect, the non-physical property is the property that is first to become epiphenomenal. This situation demonstrates the workings of causal overdetermination. If physicalism is taken seriously, then positing two causes, for one physical effect leaves one of them epiphenomenal. In the case of the mind-body problem, for Kim, physical processes would be biological processes, and their effects are observable behavior. ${ }^{26}$ Thus, Kim holds that the intrinsic nature of

\footnotetext{
21 Ibid., 15.

22 Ibid., 19-20, 33-35.

23 Ibid., 33-35, 152-153.

24 Ibid., 165, 173.

25 Ibid., 45.

26 Ibid., 23, 167.
} 
consciousness is not physically reducible, and therefore, has no causal effect on the physical world.

However, Kim states that conscious experience can be characterized functionally, given observable behaviors of conscious experience. ${ }^{27}$ Kim suggests that certain parts of the mental are reducible, such as intentionality, with intentionality referring to beliefs, desires, and perception. ${ }^{28}$ For Kim, if the intentional or cognitive aspects of the mental are functionally definable, there has to be mechanistic realizers of those functions, even if they are not all available in immediate scientific research. ${ }^{29}$ When Kim describes the functionalizability of intentionality, he outlines humans' capacity for language and the behaviors resulting from communication as an example of the mechanisms by which belief, thought, and desire can be realized. ${ }^{30}$

Kim also suggests that sensory experience, or the qualitative character of experience, can be expressed functionally. He posits that this can occur by explaining the similarity and difference relations between sensory experiences among organisms. ${ }^{31}$ For example, he holds that a sensory experience of the sight of a yellow traffic light produces similar discriminable behavior among individuals using traffic lights in most cities and towns. ${ }^{32}$ For Kim, the discriminability of sensory experiences allows for detectable behavioral differences.

\footnotetext{
${ }^{27}$ Ibid., 172-173.

28 Ibid., 5, 165-166.

29 Ibid., 164.

${ }^{30}$ Ibid., 166.

31 Ibid., 172-174.

32 Ibid., 172. 102
} 
These behavioral differences, he offers, are what are functionalizable. ${ }^{33}$ This is what Kim calls "conditional reductionism." ${ }^{34}$ Conditional reductionism means that qualitative properties are reducible to their functions, or to their causal roles, on the condition that the behavior caused by sensory experience is observable. This is to say that the mental realm is reducible, but intrinsic qualia, is not physically reducible. Kim calls this the mental residue that is left over after qualia are functionally explained. ${ }^{35}$ Kim's account addresses the problems of the non-reductivist motivations for the explanatory gap. While non-reductive intuitions coincide with conceptions of intentionality, like thoughts, desires, and beliefs as being introspectable, and providing individuals with a kind of agency, Kim demonstrates that the ineffable properties of subjective experience become epiphenomenal when faced with the task of explaining how they cause physical behavior. It is important to consider correlations with behavior and experience as explanatory of experience, I hold, because this is consistent with revising and figuring out more exact theoretical explanations of consciousness. Kim shows how the explanatory gap cannot be bridged by theories that use non-reductive, and thus epiphenomenal properties to do explanatory work.

However, materialist theories may not all coincide exactly with Kim's causal closure requirements. For example, Clark's account complicates Kim's conclusion that qualia should be defined

\footnotetext{
${ }^{33}$ Ibid., 172-173.

${ }^{34}$ Ibid., 161.

${ }^{35}$ Ibid., 170.
} 
functionally, by the roles that they perform, which is a consequence of his an argument for causal closure. I argue that despite this complication, Clark's feature-placing hypothesis is compatible with the kind of causal closure Kim sets out because on Clark's account, qualitative properties remain reducible to physical properties, despite being relationally defined, and having no relationship to the distal stimuli that Kim suggests can constitute the discriminable similarity and differences of intentionality and qualia on a functionalist account. The physical properties that qualitative properties reduce to, on Clark's account, are neural mechanisms such as feature maps and selective attention processes. Additionally, Clark's feature-placing hypothesis offers another way of individuating qualia uniquely, which is that of the spatial representation of qualitative properties. In these ways, Clark's feature-placing hypothesis can be seen as supporting a materialist explanation of qualitative properties, and suggests possibilities for bridging the explanatory gap.

\section{Austen Clark's Feature-Placing Hypothesis}

In $A$ Theory of Sentience, Austen Clark calls the mental representation of a sensory experience, feature-placing. Mental representation is the idea that when one has experiences, those experiences are about, or represent something. Feature-placing on Clark's account explains qualitative properties relationally via mental 
representation upon which subjects pick out place-times. ${ }^{36}$ Placetimes can be thought of as regions or locations in space-time. ${ }^{37}$

Picking out place-times is equivalent to having spatial discrimination abilities, or spatio-temporal-discrimination abilities. Sentient creatures possess these abilities, which act to indicate the locations of qualitative properties via the different sensory modalities. ${ }^{[38][39]}$ Clark offers that locations are picked out through a causal chain of information, where conditional probabilities between inputs and outputs determine the information channel. ${ }^{40}$ On one end of this causal chain might be feature maps, discussed more below, which are cortical regions where cells are activated, and these processes guide the behavior of the organism.

The feature-placing hypothesis defines the instances of sensations in a given sensory modality as related to one another, comparatively. Because the structure of qualitative properties are defined by their relations to one another on this account, they make no reference to stimuli or distal properties as objects. ${ }^{[41][42]}$ Clark gives the example of orange, which cannot have properties that are inherent to the color itself, but can be known as something that appears as having the character of being both reddish and yellowish. ${ }^{43}$ The

\footnotetext{
${ }^{36}$ Clark, A Theory of Sentience, 43.

37 Ibid., 41.

38 Ibid., 44.

39 Austen Clark, "Phenomenal Properties: Some Models from Psychology and Philosophy," Philosophical Issues 18 (2008): 408-409.

${ }^{40}$ Clark, A Theory of Sentience, 64, 66.

41 Austen Clark, Sensory Qualities (Oxford: Clarendon Press, 1993), 192.

42 Clark, A Theory of Sentience, 51.

43 Ibid., 12.
} 
discriminations of similarity and difference relations are among the properties of sensation that are not intrinsic, phenomenal properties. ${ }^{44}$ Rather, Clark holds that the intentional character of the sensations is nothing over and above its spatial character.

Clark demonstrates that the relational aspect between qualities can be modeled with existential quantifiers, which highlights how the features themselves are not defined by their causal role, but rather by their relations to one another. ${ }^{45}$ For example, the Ramsey sentence for red is: $\operatorname{Red}\left(\mathrm{x}_{1}\right) \equiv\left({ }_{1}\right)(2)(3)(4)(5)\left(\mathrm{C}_{12} \& \mathrm{M}_{134} \& \mathrm{R}_{145} \& \ldots ..\right)$ where $\left(\mathrm{x}_{2}\right)$ is green, $\left(\mathrm{x}_{3}\right)$ is yellow, $\left(\mathrm{x}_{4}\right)$ is orange, and $\left(\mathrm{x}_{5}\right)$ is blue. ${ }^{46} C$ stands for the complements of a particular color, and $x y$ are the color complements. Mxyz represents terms that stand in a mixing relation; for example, red and yellow mix to get orange. Rxyz describes qualities more similar to one color than another. Eventually the sentence can set equal the properties of brain states to qualitative properties. ${ }^{47}$

The Ramsey sentence, Clark says, emphasizes the interdependent nature of features. ${ }^{48}$ That is, features would not be what they are without their relations to one another. For example, the color red would not be what it is without the other qualities by which it is defined. In this way, Clark's account contrasts with functionalist accounts of qualitative properties. I maintain however, that his account is still compatible with a causal closure condition because of its reductive nature. Furthermore, functionalism might not be the only

\footnotetext{
${ }^{44}$ Ibid., 8, 11-12, 18.

${ }^{45}$ Ibid., $99,142$.

46 Ibid., 141.

${ }^{47}$ Ibid, 141-142.

${ }^{48}$ Ibid., 142. 106
} 
way to explain qualia and individuate their content. Instead, Clark holds that spatial representation can achieve both of these aims.

Clark offers that spatial representation acts to instantiate features at their spatio-temporal locations. He says that distinguishing the capacities of creatures to represent and discriminate space from the capacities individuals have to represent the features of sensation enables us to have the experience of the same quality at two different locations. ${ }^{49}$ This distinction aids in both individuating qualia, and solving the binding problem, or the problem of what allows ontologically separate features, like red, and square, to appear together as one cohesive perception. ${ }^{50}$ Multiple features can be bound together in a coherent perception because they are instantiated together at place-times. Spatial discrimination capacities of perceivers then pick out the place-times of features.

Clark also holds that feature-placing can be analogous to the feature-placing languages of P.F. Strawson, where picking out the locations of qualitative properties can act as a referent, of the designated term. ${ }^{51}$ He suggests that a causal chain from the referent to the designator happens only through perceptual encounters with the designator, and that spatial discrimination abilities account for reference. $^{52}$ In this way, the feature-placing hypothesis presents a

\footnotetext{
${ }^{49}$ Ibid., 35, 43.

50 Ibid., 39.

${ }^{51}$ Ibid., 44.

${ }^{52}$ Ibid., 76.
} 
nonconceptual alternative to postulating linguistic contents of qualitative properties. ${ }^{53}$

The reductive nature of qualitative properties can be seen through a creature's use of neural feature maps. Clark suggests that cortical regions enable spatial discrimination capacities; areas of the cortex enable perceivers to discriminate the locations of sensory qualities. ${ }^{54}$ Clark holds that the cells of cortical regions corresponding to the sensory modalities can detect the incidence of basic features. This then leads to the detection of the location of qualitative properties. For example, in the auditory modality, detecting frequencies can enable perceivers to detect distances and other spatial features. ${ }^{55}$ The activation of cells in areas of the cortex enables a causal chain of the primitive detection of qualitative properties. ${ }^{56}$

\section{Feature maps, place-coding, and selective attention}

The mechanisms in the cortex that detect the locations of features are called feature maps. Feature maps are topographically organized and give a sensation a local region of occurrence. ${ }^{57}$ For example, feature maps representing in the visual cortex start with cells being able to make discriminations of edges, which will also be the discrimination of the locations of edges. Edges of colors, for example, are processed at this early stage of perception. ${ }^{[58][59]}$ Processing the

\footnotetext{
53 Austen Clark, "Sensing, Objects, and Awareness," 578.

54 Clark, "How Do Feature Maps Represent?"

55 Clark, "How Do Feature Maps Represent?"

${ }^{56}$ Clark, A Theory of Sentience, 62.

${ }^{57}$ Clark, "Location, Location, Location," 293.

58 Clark, "Feature-Placing," 443. 108
} 
locations of edges comes early in the hierarchy of perception because the edges are where the discriminable differences lie. This then, leads to spatial-discrimination abilities. ${ }^{60}$ Clark holds that feature maps can represent distal features and their locations, and this is the process of feature-placing. ${ }^{61}$

One way of getting from cells firing in the cortex to the locations of features is through place-coding. Place-coding links the information in feature maps to information that enables the locations of qualitative properties to be identified. The way this works is through the receptive fields of the nerves in a modality. They correspond to the activation patterns of neurons in the cortex. The location of receptive fields might correspond to a "pool" of neurons, as Clark says, that carry information about the location of the receptive field of the nerves. ${ }^{62}$ Clark gives an example of locating an itch: the pools of neurons in the somatosensory cortex correspond to distinct receptive fields on the skin, so when one pool activates, it carries information about where its receptive field is, and thus where the itch is located. ${ }^{63}$ In this way, the locations of sensation can be discerned with information from feature maps.

The feature-placing hypothesis sets out to explain only primitive sensory representation, since the processes feature-placing accounts for happen in the earliest stages of sensory experience. As feature-placing processes are the earliest stage of conscious

\footnotetext{
${ }^{59}$ Clark, A Theory of Sentience, 134-135.

${ }^{60}$ Clark, "Location, Location, Location," 295.

${ }^{61}$ Ibid., 294.

${ }^{62}$ Clark, A Theory of Sentience, 96.

${ }^{63}$ Ibid.
} 
perception, feature maps that create representations identifying the incidence of features do not identify objects, or individuate them. ${ }^{64}$ In this way, feature-placing is an unconscious process on the part of perceivers. $^{65}$ Clark maintains that following from this, that the contents of qualitative properties are nonconceptual, and prelinguistic. ${ }^{66}$ Early-content sensory processes lack object individuation, and recognition by the sentient individual of such processes. ${ }^{[67][68]}$ For Clark, perceptual experience occurs in stages, the first of those being nonconceptual, and pre-attentive, or unconscious.

Accordingly, Clark argues in favor of the spotlight of attention, or selective-attention model, which is consistent with the idea that organisms process sensory content preattentively. ${ }^{69}$ The spotlight of attention theory, or selective attention model has it that the central processing system of a creature selects information from incoming peripheral processing. That information then falls into the attention of the organism, or is spotlighted. Clark states, "Selective attention is thought to be a process that selects some representations

${ }^{64}$ Clark, "How Do Feature Maps Represent?"

${ }^{65}$ Austen Clark, "Sensory and Perceptual Consciousness," in The Blackwell Companion to Consciousness, ed. Max Velmans and Susan Schneider (Hoboken: Blackwell Publishing Ltd., 2007), 446-447.

${ }^{66}$ Clark, A Theory of Sentience, 80.

${ }^{67}$ Ibid., 83.

${ }^{68}$ Austen Clark, "Feature-Placing and Proto-Objects," Philosophical Psychology 17 (2004): 466.

${ }^{69}$ Austen Clark, "Color, Qualia, and Attention," in Color Ontology and Color Science, ed. Jonathan Cohen and Mohan Matthen (Cambdridge: MIT Press, 2010), http://selfpace.uconn.edu/paper/ClarkAusCQAtt.pdf, page 11. 
but not others for further "central' processing."70 Clark iterates that basic features, on the hierarchical model of perception, are processed preattentively, and selective attention stands as the transition point between preattentive stages of perception, and the attentional awareness stage of perception. ${ }^{71}$ Features that are being processed simultaneously and in parallel, on Clark's account are in a sense, in competition for selection. ${ }^{72}$ When they are selected, they surpass preconscious processing into the state in which a subject has awareness of what she is perceiving. ${ }^{73}$

The mechanisms of selective attention are also consistent with content that is processed nonconceptually. Because receptors taking in preattentive processes cannot be turned off, as Clark says, parallel, peripheral processes are selected by mechanisms that highlight the information that eventually becomes conscious. ${ }^{74}$ In this way, preattentive processes are those that happen without a subject being consciously aware of these processes. Selective attention mechanisms allow for the possibility of distinguishing between the qualia, or the features themselves, and the awareness one has of perceiving those qualia. $^{75}$ During preattentive perception, one is processing the basic

\footnotetext{
70 Austen Clark, "Cross-Modal Cueing and Selective Attention" in The Senses: Classical and Contemporary Philosophical Perspectives, ed. Fiona Macpherson (Oxford \& New York: Oxford University Press, 2011), 376.

71 Austen Clark, "Perception Preattentive and Phenomenal," in Philosophy of Psychology and Cognitive Science, ed. Paul Thagard (New York: North Holland/Elsevier, 2007), 167.

72 Ibid., 168.

73 Clark, "Color, Qualia, and Attention," 2.

74 Ibid., 10.

${ }^{75}$ Ibid., 9-10.
} 
qualia, while after those features are spotlighted, one can become aware of what is being perceived.

As this sort of bottom-up processing theory of perception entails, the feature-placing hypothesis will have it that these processes eventually lead to the conscious awareness of an organism. However, feature-placing works in a layered manner, starting with early, unconscious content, and does not refer to conscious awareness. ${ }^{76}$ In fact, Clark holds that affixing the notion of conscious awareness to all properties of the mental suggests that creature-consciousness is consistently subjectively veridical. ${ }^{77} \mathrm{He}$ suggests that this paradigm creates a bias metaphysics for consciousness in that he states that this allows for appearances to generate reality. ${ }^{78}$ He holds that considering subjective experience to be fundamental gives the mental realm a metaphysical freedom that does not apply to explanations of phenomena in the physical sciences. ${ }^{79}$

Because the sensory content of feature-placing occurs nonconceptually, and because qualitative properties can be reduced to feature maps on Clark's account, I hold that the feature-placing hypothesis meets a causal closure condition, and this supports a materialist account of mental phenomena that can help to bridge the explanatory gap. The aspect of Clark's account that defines qualitative properties relationally, without reference to a physical cause, or to objects, presents a problem for functional accounts that tie

\footnotetext{
76 Clark, A Theory of Sentience, 88, 93.

77 Clark, "Phenomenal Properties," 421, 423.

78 Ibid., 423.

${ }^{79}$ Ibid., 422-423. 112
} 
explanations of behavior (for example, psychoneural or biological behavior) to the stimuli that caused the behavior. The motivation for such an account was seen through the work of Kim, above.

Despite the incompatibility of Clark's feature-placing hypothesis with the notion that reference to stimuli allows for a functional description of behavior, I hold that it remains compatible with a causal closure condition because qualitative properties, on the feature-placing hypothesis, are to be reduced to physical properties. Clark's relational qualitative properties, and the postulated spatial discrimination abilities that organisms have to locate and discern those properties, together, provide a plausible answer to what individuates qualitative properties. For this reason, I think feature-placing can do work in providing an explanation that bridges conscious states with physical properties.

Kim's account of mental causation and Clark's account of feature-placing both suggest that qualia, if it is identified with intentionality, or conceptual content all-the-way-down will engender an impossible and impoverished analysis of the ontological nature of qualia. Clark and Kim seem to agree on one thing: that the intrinsic, ineffable nature of qualia has no causality in the physical world. [80][81] Clark's feature-placing model is one way to achieve and represent the physicalism-near-enough that Kim puts forward, and that the causal closure of the physical world requires. In this way, the explanatory gap might be bridged from a materialist's perspective because the sensory properties of mental qualia are explained relationally, and

\footnotetext{
${ }^{80}$ Ibid., 423-424.

${ }^{81}$ Kim, Physicalism or Something Near Enough, 173-174.
} 
those properties are physically reducible to spatial discrimination capacities.

\section{Conclusion}

Where Clark's feature-placing hypothesis addresses qualitative properties relationally, and identifies and individuates their content through spatial representation, the account only extends to primitive sensory content, and so to some advocates of the explanatory gap, might not address subjective experience completely. However, I offer here that this is the best characterization of qualitative properties, in so that they do not become epiphenomenal. For those who desire a theory of consciousness consistent with the completeness of physics, or an ontological naturalism, the view that qualitative properties are intrinsic aspects of subjective experience will most likely not bridge any gaps in explanation between consciousness and physicalism because these properties are nonreductive, so it is hard to see how they remain causally effective upon conscious experience. In this way, the subjective experience of Nagel and the problems with physicalism in explaining consciousness suggested by Levine will be epiphenomenal properties of experience, if the causal closure of the physical world is correct.

Clark's feature-placing hypothesis fits nicely with an ontological naturalism and causal closure, and extends the principles of materialism to concepts about the phenomenal. I offer that Clark's feature-placing hypothesis also remains a viable candidate for the physical reduction of qualitative properties because it allows for nonconceptual, preattentive content, which bolsters its compatibility 
with a causal closure condition. This sort of account seems to provide some kind of bridge in explanation between consciousness and physicalism.

A psychophysical explanation of sensory experience, (one that connects perceptual states to the effects of the physical states of perceivers) such as feature-placing, provides an empirical basis to build more elaborate and exact theories of conscious experience. Describing our capacities to pick out features by spatially discriminating them allows for an objective characterization of "what it's like," as Clark says. ${ }^{82}$ This approach seems to make room for further empirical discoveries and hypotheses about qualitative properties that also aim at bridging the explanatory gap.

\section{References}

Clark, Austen. A Theory of Sentience. Oxford: Oxford University Press, 2000.

—: "Color, Qualia, and Attention," in Color Ontology and Color Science, edited by Jonathan Cohen and Mohan Matthen. Cambridge: MIT Press, 2010.

—: "Cross-Modal Cueing and Selective Attention." In The Senses:

Classical and Contemporary Philosophical Perspectives, edited by Fiona Macpherson. Oxford \& New York: Oxford University Press, 2011. 375- 396.

-: "Feature-Placing and Proto-Objects." Philosophical Psychology 17 (2004): 443-469.

\footnotetext{
${ }^{82}$ Clark, Sensory Qualities, 207.
} 
—: "How do feature maps represent?" Paper presented at the Early Content Conference, College Park, April 22, 2006. http:// selfpace.uconn.edu/paper/ClarkAusUmEarly.html.

—: "Location, Location, Location." In Computation, Cognition, and Pylyshyn, edited by Don Dedrick and Lana Trick. Cambridge: The MIT Press, 2009. 281-302.

—: "Perception Preattentive and Phenomenal." In Philosophy of Psychology and Cognitive Science, edited by Paul Thagard. New York: North Holland/Elsevier, 2007. 159-193.

—: "Phenomenal Properties: Some Models from Psychology and Philosophy.” Philosophical Issues 18 (2008): 406-425.

—: "Sensing, Objects, and Awareness: Reply to Commentators." Philosophical Psychology 17 (2010): 553-579.

—: "Sensory and Perceptual Consciousness," in The Blackwell Companion to Consciousness, edited by Max Velmans and Susan Schneider. Hoboken: Blackwell Publishing Ltd., 2007. 445-455.

—: Sensory Qualities. Oxford: Clarendon Press, 1993. Jackson, Frank. "Epiphenomenal Qualia.” In Mind and Cognition: A Reader, edited by William G. Lycan (Basil Blackwell, 1990), 469-477.

Kim, Jaegwon. Physicalism or Something Near Enough. Princeton and Oxford: Princeton University Press, 2005.

—: "From Naturalism to Physicalism: Supervenience Redux." Proceeding and Addresses of the American Philosophical Association 85 (2011): 109-134. 
Levine, Joseph. "On Leaving Out What It's Like.” In The Nature of Consciousness: Philosophical Debates, edited by Ned Block,

Owen Flanagan, and Güven Güzeldere. Cambridge: The MIT Press, 1997. 543-555.

Nagel, Thomas. "What Is It Like to Be A Bat?" The Philosophical Review 83 (1974): 435-450. 